\title{
An Automatic Level Set Based Liver Segmentation from MRI Data Sets
}

\author{
Evgin Goceri ${ }^{1}$, Mehmet Z. Unlu ${ }^{2}$, Cuneyt Guzelis ${ }^{3}$ and Oguz Dicle ${ }^{4}$ \\ ${ }^{1}$ Computer Engineering Department, Pamukkale University, Kınıkl1, Denizli, Turkey \\ e-mail: egoceri@pau.edu.tr \\ ${ }^{2}$ Electrical and Electronics Engineering Department, İzmir Institute of Technology, Urla, İzmir, Turkey \\ e-mail: zubeyirunlu@iyte.edu.tr \\ ${ }^{3}$ Electronics and Telecommunications Engineering Department, İzmir University of Economics, Balçova, İzmir, Turkey \\ e-mail: cuneyt.guzelis@izmirekonomi.edu.tr \\ ${ }^{4}$ Faculty of Medicine, Radiology Department, Dokuz Eylül University, Narlıdere, İzmir, Turkey \\ e-mail: oguz.dicle@deu.edu.tr
}

\begin{abstract}
A fast and accurate liver segmentation method is a challenging work in medical image analysis area. Liver segmentation is an important process for computer-assisted diagnosis, pre-evaluation of liver transplantation and therapy planning of liver tumors. There are several advantages of magnetic resonance imaging such as free form ionizing radiation and good contrast visualization of soft tissue. Also, innovations in recent technology and image acquisition techniques have made magnetic resonance imaging a major tool in modern medicine. However, the use of magnetic resonance images for liver segmentation has been slow when we compare applications with the central nervous systems and musculoskeletal. The reasons are irregular shape, size and position of the liver, contrast agent effects and similarities of the gray values of neighbor organs. Therefore, in this study, we present a fully automatic liver segmentation method by using an approximation of the level set based contour evolution from $\mathrm{T} 2$ weighted magnetic resonance data sets. The method avoids solving partial differential equations and applies only integer operations with a two-cycle segmentation algorithm. The efficiency of the proposed approach is achieved by applying the algorithm to all slices with a constant number of iteration and performing the contour evolution without any user defined initial contour. The obtained results are evaluated with four different similarity measures and they show that the automatic segmentation approach gives successful results.
\end{abstract}

Keywords-Liver segmentation, MRI, Geometric active contours, Level set method

\section{INTRODUCTION}

The use of Magnetic Resonance (MR) images for liver segmentation has been limited when we compare MR applications in central nervous systems and musculoskeletal. Since liver that is the largest organ requires a large field of view. Therefore, the available spatial resolution for liver is less than the resolution with the field that is used for brain and joint imaging. Another significant handicap to use MR images for liver segmentation is motion artifacts. Also, Computer Tomography (CT) is less costly than MR imaging. [1]. However, the concerns about the radiation risks and also the iodinated contrast agents associated with abdominal CT images are increasing [2].

MR imaging, which uses non-ionizing radiation, has become the dominant clinical imaging modality and it is considered as the cornerstone of abdominal imaging especially for liver organ with recent progress in image acquisition techniques, digital image processing and computer technology. It is now possible to overcome the motion artifacts by using several breath-hold sequences, cardiac gating, respiratory, and different motion suppression techniques. Also, images in high resolution within a single breath-hold can be obtained by efficient changes of pulse sequence design and faster switching mechanisms of gradient amplifiers. More powerful gradients and more sensitive receiver coils give images with improved spatial resolution. In addition, purpose-built applications can be developed by using different MR imaging modalities.
Although, developments in technology have improved the quality of liver MR images, liver segmentation task from MR images is still very challenging. One important reason is that the acquisition of good quality images requires an unflagging attention to details of technique. [1]. Another reason is irregular shape and position of the liver (Fig.1). Also, gray level similarities between the liver and neighbor organs (Fig.1. $a$ and $b$ ) make the liver segmentation from MR data sets more difficult. Different contrast agents, which have important diagnostic value for liver MR imaging, are used in many cases even MR images have superior contrast resolution. However, the use of contrast agents may causes all tissues to have varied gray values in each MR images and even in each slices. The contrast agent effect increases these challenges of liver segmentation from MR images. Therefore, liver segmentation studies are mostly in CT images in the present literature [3-8].
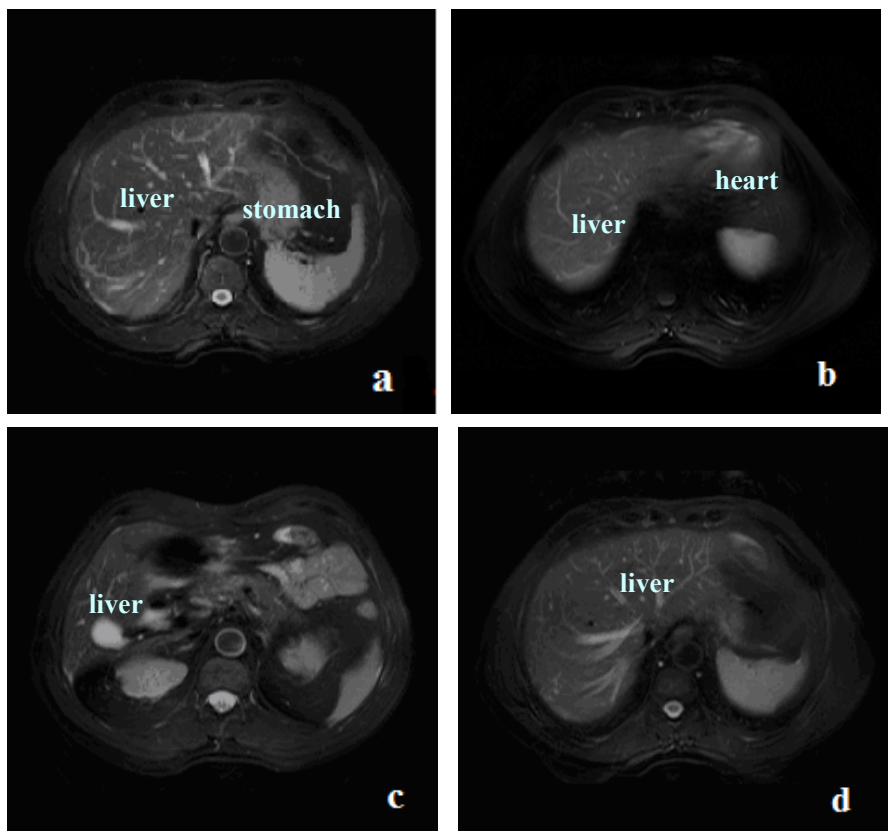

Fig.1. Examples of abdominal MR images and representation of difficulties for liver segmentation: Similar gray level values between liver and stomach (a), liver and heart (b), a slice from the beginning (c), and a slice from the end of the same MR data set to represent irregular shape of liver $(\mathrm{d})$ 
There exist only a few studies in the literature for the liver image segmentation from MR data sets, which have been proposed by using snakes [9], fast marching method [10], feed forward neural network [11], fuzzy c-means based segmentation [12,13], graph-cut approach [14], synchronized oscillator network [15], active shape model [16,17], watershed [18], iterative quadtree decomposition method [19], Gaussian model and markov random field [20], modified region growing [21], and free form registration on manually segmented CT images [22]. Some of these methods are time consuming and have complex calculations such as active contour based approach $[9,10$ $16,17,22]$ or the used MR image modality characteristics are not clearly identified $[11,15,18]$. Statistical shape model based and statistical gray level value distribution based methods [20] need both prior shape knowledge of the liver and huge number of training data set.

Two different level set based liver segmentation methods have been proposed by using MR data sets in the literature. The idea behind the level set method is to evolve an initial contour over an image towards the desired boundaries. One of the proposed level set based method for liver segmentation is a semiautomatic segmentation approach [23] that is very time consuming due to the calculations with Partial Differential Equations (PDEs) and needs extra time for initializations by users. Also, different users initialize images differently which leads to different computation time and results. The other level set based method [24] requires prior shape and size knowledge of the liver and a huge number of training data set. The problems of different modality characteristics, atypical liver shapes and similarities of gray values of neighbor tissues are not solved by using these methods. Therefore, these methods do not deal with all the problems at the same time and liver segmentation using MR images is still problematic. In this study, we propose an automatic and iterative liver segmentation approach that uses an approximation of the level set method [25] to overcome these difficulties.

The organization of the paper is as follows. The properties of the MR data sets used are given in Section II. Basics of active contours, level set method and related works are presented in Section III. We explain the proposed liver segmentation method in Section IV. Finally, we present the experimental results and conclusions in Section V.

\section{DATA SET}

The upper abdominal MR data sets have been obtained from three different patients using a 1.5 Tesla MR imaging device (Gyroscan Intera, Philips, ACS-NT, Best, The Netherlands) located in Dokuz Eylül University Radiology Department. The examined 16 bit DICOM images are fat suppressed T2-weighted (TR/TE, 1600/70 ms; flip angle, $90^{\circ}$; slice thickness, $8 \mathrm{~mm}$ ) SPIR images in the axial plane with a resolution of $256 \times 256$.

\section{ACTIVE CONTOURS FOR IMAGE SEGMENTATION}

Active contour techniques are widely used for image segmentation. Several reviews about active contour methods have been published [39-41].

Parametric active contours, which are snakes, introduced by Kass et al. (1988) [42], use an energy minimization approach to segment images. A given initial contour is evaluated by minimizing its energy that is derived from the active contour as internal energy and from the image as external energy. Parametric active contours can not handle topological changes. Also, the magnitude of the external forces become smaller very quickly while evolving from the image boundaries which causes small capture range of snakes. Therefore, variations of the parametric active contour method have been proposed [43-50] by using edge-based or region-based external forces to handle topological changes or shape-based prior knowledge to avoid spurious edges.

Geometric active contours, which were proposed by V. Caselles et al. [29] and R. Malladi et al. [30], use both image data and geometric information of the evolving contour for image segmentation. Geometric active contours are based on the level set approach [26] and the theory of curve evolution [51] which uses PDEs.

The importance of PDEs has increased in image segmentation area since a segmentation problem can be transformed into a PDE framework and finite difference methods can be used to solve PDEs. In addition, PDEs can be extended from 2-D to higher dimensions and allow to perform image segmentation methods with user interaction. The PDE for the deformation of the contour is written as,

$$
\frac{\partial C}{\partial t}=F(\kappa) \vec{N}
$$

that evolves the contour $C$ by the time $t$ in the normal vector direction $N$ with the speed function $F(\kappa)$, which depends on the curvature of the contour. The contour propagates faster in a high curvature area than a low curvature area.

The level set method uses the solution of a Hamilton-Jacobi equation of the higher dimensional smooth level set function $\phi$ for the contour evolution. An important advantage to use the Eulerian Hamilton-Jacobi equation is that the given level set function always remains as a function as long as the speed function is smooth [26]. The contour $C(s, t)$ is embedded as the zero level set of the function $\phi(x, y, t)$ as,

$$
\phi(C(s, t), t)=0
$$

and evaluated towards the desired object boundaries. The motion equation is obtained by differentiating (2) with respect to time $t$ and the level set equation is written by using (1) as,

$$
\frac{\partial \phi}{\partial t}+F(\kappa)|\nabla \phi|=0
$$

Improved level set methods have been proposed to handle topological changes and to obtain accurate results from image segmentation applications [52-55,31-33,35-38,40,27,28]. An energy functional is used to obtain an evolution PDE and energy minimization is applied in the improved level set methods. For instance, local intensity information has been incorporated to the level set method as a solution for intensity inhomogeneity problem in [35$38,27]$. However, only mean values of local intensities do not enough for accurate segmentation when there exists severe noise and intensity inhomogeneity. Therefore, both mean values and variance values of local intensities, which are obtained by Gaussian distributions, have been used to deal with intensity inhomogeneity in [34]. Although, this approach has not been applied yet for liver images, it gives accurate results for brain images but causes high computational cost. The piecewise constant model based level set method in [56] can not handle intensity inhomogeneity problem. Only region-based features have been used in [28] by assuming the existence of only object that will be segmented and background in the image. However, the used global information in the proposed formulation is not accurate when there is intensity inhomogeneity in inside or outside the contour. The level set method that uses a shape based prior knowledge in $[52,54,55]$ and the supervised segmentation method in [53] needs prior information. 
Also, the narrow banded level set methods in [67-60] have been proposed to decrease calculation time by increasing the curve evolution speed. The evolution of the curve is performed by solving the PDEs only around the neighborhood of the zero level set instead of in the whole image in these methods.

\section{PROPOSED METHOD FOR LIVER SEGMENTATION}

In this study, a new fully automatic liver segmentation approach from SPIR data sets is presented by using the Fast Two-Cycle (FTC) algorithm [25] that enables an initial contour evolution with two different cycles. This algorithm approximates the level set method without solving PDEs and reduces the computational cost. This is a narrow band method with a bandwidth of two pixels. The data dependent evolution is performed in the first cycle and the curve smoothing operation is performed in the second cycle. The contour evolution is represented with two linked lists, which contain the points inside the contour and the points outside the contour, by using only integer values. The evolution in both cycles is achieved by a switching mechanism of elements between these two lists. A data dependent speed function is used for evolution in the first cycle. A curve dependent speed function is used in the second cycle for smoothness regularization. The value of the smoothing speed is obtained from Gaussian filtering. The final values of these speed functions are -1 , or 0 , or +1 after thresholding process. If the curve evolution is inward then the sign of the speed functions is negative. If the evolution is outward then the sign of the speed functions is positive.

Initial contours can be moved outward or inward and their position, which is a key challenge for level set based segmentation methods, determines the segmentation result [61]. The drawback of the Shi and Karl's level set method [25] is its sensitivity of initial contours. Because, the accuracy of the obtained results mainly depends on the number, size and location of the initial contour that are drown by users. Also, the given iteration number for each slice affects the results. Therefore, this approach is not robust and can generate over segmented or under segmented images. In order to overcome this drawback, we applied this method iteratively without any user interaction and with a small constant number of iterations for liver segmentation from SPIR data sets.

In our strategy, the initial contour for each slice is obtained by using the relation between sequential slices in a dataset. The liver shape in a slice is close to the liver shape of the previous or next slice than other slices. Therefore, the shape of an initial liver image segmented formerly is used as the initial contour for the consecutive slices.

The initial liver image is obtained from the selected initial slice (Fig.2.a) in which the border of liver is very clear in the dataset. It is usually the mid-slice of datasets, which comes just after the first disappearance of the right kidney because the liver boundaries do not overlap with other boundaries of neighbor organs and used as the initial slice to be segmented.

To obtain the initial liver image, the selected initial slice is clustered by applying the most commonly known unsupervised clustering method K-means, which gives a successful result only for the selected initial slice. Then, the cluster that has the liver organ is selected from the three clusters that are background, dark regions (skin) and bright regions (organs) (Fig.2.b). Binary morphological operations are applied to the selected cluster after the clustering step. The first binary operation is erosion to remove the weakly connected objects (Fig.2.c). Then, a connected component analysis is performed to extract the biggest part (Fig.2.d). Next operation is dilation to restore the completely undeleted objects by erosion (Fig.2.e). Finally, filling is applied for holes in the image and the segmented initial liver image (Fig.2.f) is obtained to use its shape as the initial contour for the first succeeding and preceding slices.
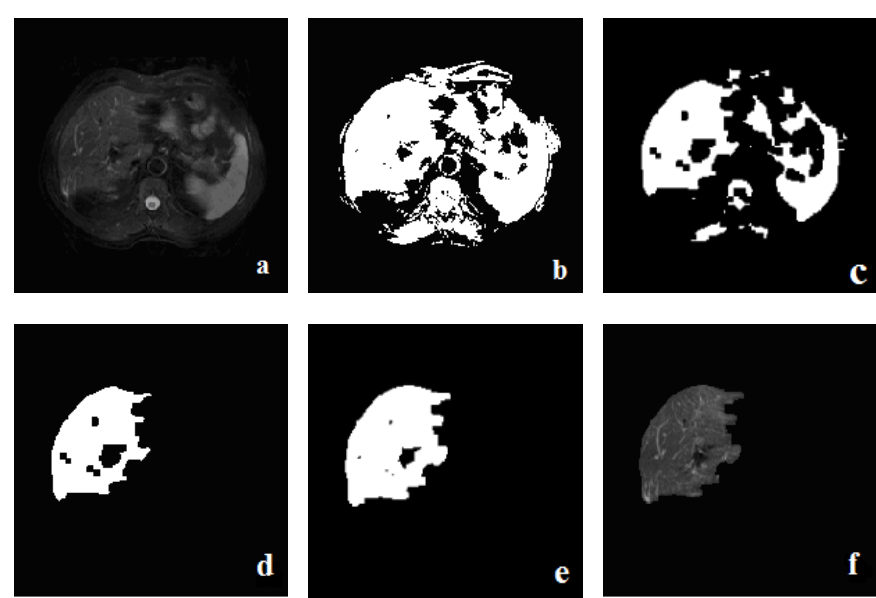

Fig.2. Initial liver image segmentation: Selected initial slice (a), clustered image (b), after erosion (c), the biggest part (d), after dilation (e), initial liver image (f)

After obtaining the initial liver image, each preceding and succeeding slices are segmented iteratively with the same number of iteration, which is a very small number (only 10 iterations for each slice) because the initial contour for each slice is near the desired liver edges. Therefore, the required time for the segmentation of each slice is reduced and the segmented images are obtained automatically from all slices in the data set.

A series of binary morphological operations are applied to the segmented liver images to remove mis-segmented regions in the postprocessing stage. These operations are the same with the steps that are used to obtain the initial liver image from the clustered image.

\section{RESULTS AND CONCLUSIONS}

Figure 3 shows that the results obtained by the proposed liver segmentation approach are successful.

K-means, which is a commonly known segmentation method, gives unsuccessful results for liver segmentation from MR images. Example results of K-means based liver segmentation by using our data set are shown in Fig.3.
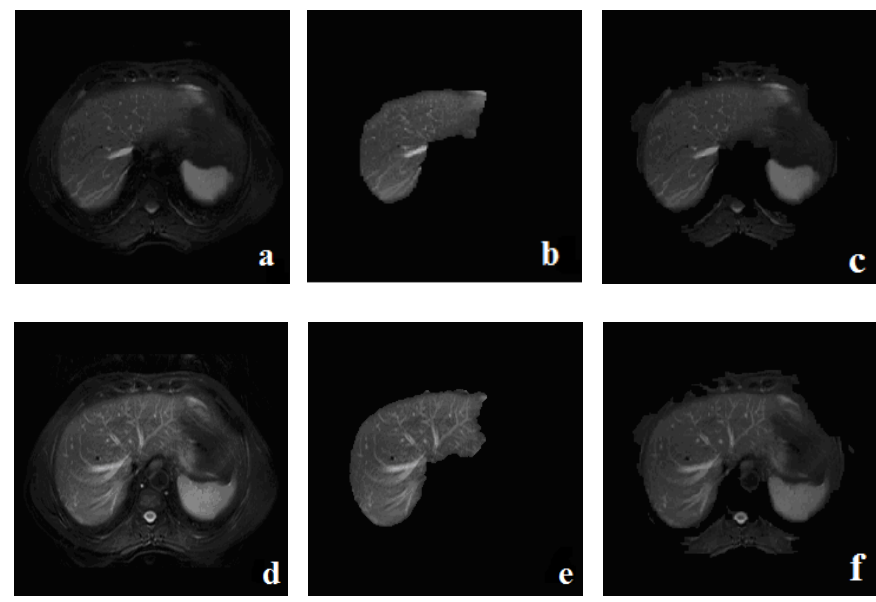

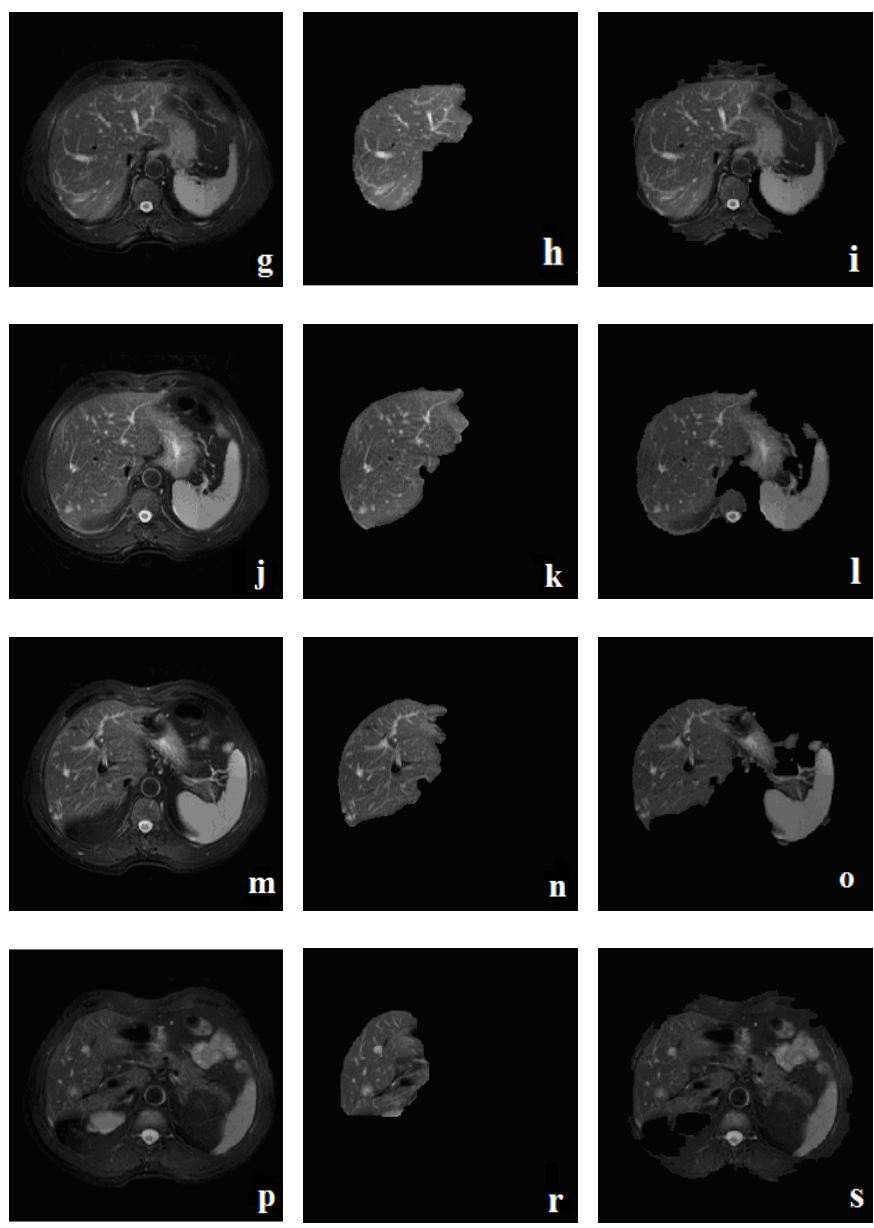

Fig. 3. Original slices (a,d,g,j,m,p); Level set based segmentation results (b,e,h,k,n,r); K-means based segmentation results (c,f,i,l,o,s)

The quantitative analysis for performance comparison between level set based segmentation results and their reference images has been evaluated by using four different similarity measurements. The first similarity metric is Hausdorff distance [63] which is a maximum surface distance. Because it computes the maximum distances of pixels between their corresponding edge points and formulated by

$$
\max _{i, j}\left(d\left(x_{i}, Y\right), d\left(y_{j}, X\right)\right)
$$

where $X$ is the set of data points of a reference image $X=\left\{x_{1}, x_{2}, x_{3}, \ldots ., x_{N x}\right\}$ and $Y$ is another set of data points of a segmented image $Y=\left\{y_{1}, y_{2}, y_{3}, \ldots . ., y_{N y}\right\}, d$ is the Euclidean distance, which calculates the distance from a pixel $x_{i}$ to the point set $Y$ (and vice versa), given as $d(x, y)=\sqrt{\left(x_{1}-y_{1}\right)^{2}+\left(x_{2}-y_{2}\right)^{2}}$ These distances are computed by

$$
d\left(x_{i}, Y\right)=\min _{y_{j} \in Y}\left\{d\left(x_{i}, y_{j}\right)\right\}
$$

and

$$
d\left(y_{j}, X\right)=\min _{x_{i} \in X}\left\{d\left(y_{j}, x_{i}\right)\right\}
$$

Another metric is the Mean Squared Error Distance (MSED) [67], which is calculated by

$$
\frac{1}{M N} \sum_{i=1}^{M} \sum_{j=1}^{N}\|X(i, j)-Y(i, j)\|^{2}
$$

where $X(i, j)$ is a reference image and $Y(i, j)$ is a segmented image. If the MSED value is zero then the result is perfect, which means the segmented and the reference images are the same. Generally, the MSED is converted to a Peak Signal to Noise Ratio (PSNR) measure which is given by

$$
\log _{10} \frac{d^{2}}{M S E D}
$$

where $d^{2}$ shows the range of intensity values. Our images are 16bit/pixel so $d=2^{16}-1=65535$.

The last used similarity criteria is the Dice similarity [62,25], which is the twice intersection (overlap) of the segmented and the reference volume divided by the sum of the segmented and the reference volume, formulated by

$$
\frac{2(X \cap Y)}{X+Y}
$$

If this measurement is equal to one then there is not undersegmentation and over-segmentation and if it is equal to zero then the regions are completely different.

The quantitative analysis has been performed by using the results in Fig. 3 and the manually segmented reference liver images shown in Fig. 5 .
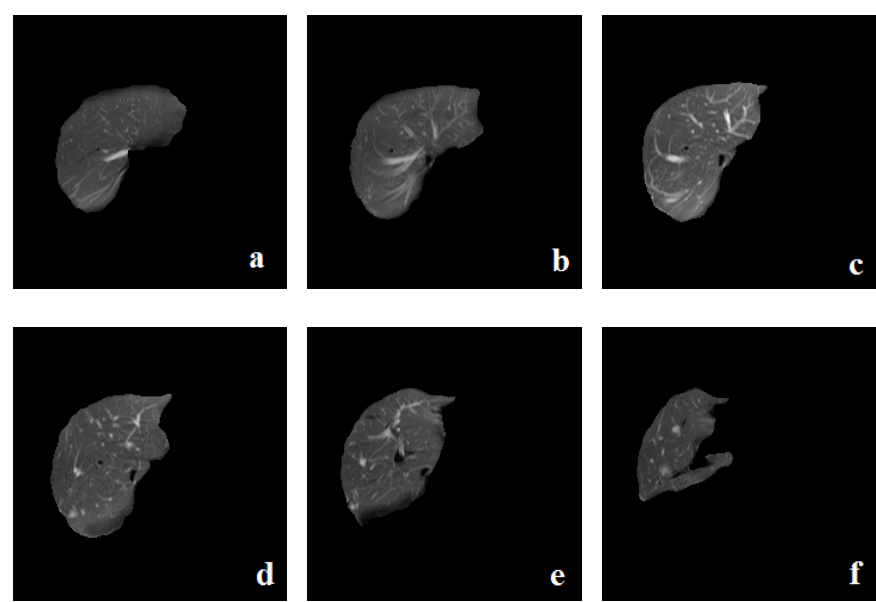

Fig.5. Manually segmented reference images (a,b,c,d,e,f) of the original slices in Fig.3.a,d,g,j, $m$ and $p$ respectively

Table 1 presents the coefficients of the four similarity metrics for the results (Fig.3.b,e,h,k,n,r) obtained from the proposed automatic liver segmentation method. The required segmentation time in seconds of all slices for this method is 13.9648 by using 2 GB RAM and $2.33 \mathrm{GHz}$. Intel Pentium CPU. 
Table 1 . The similarity values for the proposed level set based segmentation results shown in Fig. 3 using the reference images in Fig..5

\begin{tabular}{|l|c|c|c|c|}
\hline Slice & $\begin{array}{c}\text { Dice } \\
\text { coefficient }\end{array}$ & $\begin{array}{c}\text { MSED } \\
\text { coefficient }\end{array}$ & $\begin{array}{c}\text { Hausdorff } \\
\text { coefficient }\end{array}$ & $\begin{array}{c}\text { PSNR } \\
\text { coefficient }\end{array}$ \\
\hline Fig.3.b & 0.9226 & 17.8171 & 14.4222 & 18.8860 \\
\hline Fig.3.e & 0.9451 & 5.2550 & 7.2111 & 20.5833 \\
\hline Fig.3.h & 0.9318 & 12.1277 & 12 & 19.1393 \\
\hline Fig.3.k & 0.9170 & 15.5977 & 14.1421 & 18.0364 \\
\hline Fig.3.n & 0.9210 & 12.6358 & 10.6301 & 19.0161 \\
\hline Fig.3.r & 0.8829 & 31.8287 & 18.0278 & 17.6765 \\
\hline
\end{tabular}

A good overview on medical image segmentation methods using active contours is presented in [65]. Both qualitative and quantitative comparison results of eight different active contour methods except the application specific methods are presented by the authors for brain MRI, ultrasound pig heart image, kidney CT image, knee MRI, and microscopy blood cell image. However, to our knowledge, there is no liver segmentation method from T2 weighted SPIR images. There is only one study, which is kidney segmentation, that uses SPIR datasets [66]. We have preferred to use SPIR images for liver segmentation due to their clear visualization of the vascular structure that is used to separate the liver into anatomically meaningful segments and gives important information to surgeons. The quantitative and qualitative analysis show that our strategy deals with all difficulties for liver segmentation and gives successful results in a short time. The efficiency of the proposed fully automatic liver segmentation method is achieved by applying the FTC algorithm to all slices iteratively without using any user defined initial contour.

\section{REFERENCES}

[1] P. J. A. Robinson and J. Ward. MRI of The Liver - A Practical Guide. Published by Taylor \& Francis Group, ISBN: 978-0-8247-2871-7, 2006

[2] D. J. Brenner and C.D. Elliston. Estimated Radiation Risks Potentially Associated with Full Body CT Screening. Radiology, 232:735-738, 2004

[3] M. A. Selver, A. Kocaoğlu, G. K. Demir, H. Doğan, O. Dicle and C. Güzeliş. Patient Oriented And Robust Automatic Liver Segmentation For PreEvaluation Of Liver Transplantation. Computers in Biology and Medicine, $38: 765-784,2008$

[4] K. Suzuki, R. Kohlbrenner, M. L. Epstein, A. M. Obajuluwa, J. Xu and M. Hori. Computer-Aided Measurement Of Liver Volumes In CT By Means Of Geodesic Active Contour Segmentation Coupled With Level-Set Algorithms. Med. Phys., 37(5):2159-2166, 2010

[5] X. Wang, C. Zheng, C. Li, Y. Yin and D. D. Feng. Automated CT Liver Segmentation Using Improved Chan-Vese Model with Global Shape Constrained Energy. 33rd Annual International Conference of the IEEE EMBS Boston, Massachusetts USA, 2011

[6] M. G. Linguraru, J. K. Sandberg, Z. Li, F. Shah and R. M. Summers. Automated Segmentation And Quantification of Liver And Spleen From CT Images Using Normalized Probabilistic Atlases And Enhancement Estimation. Med. Phys.,37(2):771-783, 2010

[7] M. Goryawala, M. R. Guillen, M. Cabrerizo, A. Barreto, S. Gulec, T. C. Barot, R. R. Suthar, R. N. Bhatt, A. Mcgoron and M. Adjouadi. A 3D Liver Segmentation Method with Parallel Computing for Selective Internal Radiation Therapy. Information Technology in Biomedicine, IEEE Transactions, 16(1):62-69, 2012

[8] C. Xinjian, J. K. Udupa, U. Bagci, Y. Zhuge and J. Yao. Medical Image Segmentation by Combining Graph Cuts and Oriented Active Appearance Models. IEEE Trans. on Image Proc., 21(4), April 2012

[9] C. Krishnamurthy, J. J. Rodriguez and R. J. Gillies. Snake-Based Liver Lesion Segmentation. IEEE, In Southwest04, 187-191, 2004

[10] S. Nowozin and G. Lixu. A novel Liver Perfusion Analysis Method. IEEE Engineering in Medicine and Biology 27th Annual Conf., Shanghai, China, 3063-3066, 2005

[11] W. A. Gibby. Basic Principles of Magnetic Resonance Imaging. Neurosurg Clinics of North America, 16:1-64, 2005

[12] V. Positano, B. Salani, B. Scattini, M. F. Santarelli, A. Ramazzotti, A. Pepe, M. Lombardi, L. Landini. A Robust Method for Assessment of Iron
Overload in Liver by MRI. IEEE, Eng. in Medicine and Biology Conf., 2895 2898,2007

[13] D. Lu, J. Zhang, X. Wang, J. Fang. A Fast and Robust Approach to Liver Nodule Detection in MR Images. IEEE, Bioscience and Info. Tech. Conf., Jeju City, South Korea, May 2007

[14] L. Massoptier and S. Casciaro. Fully Automatic Liver Segmentation Through Graph-Cut Technique. IEEE EMBS $29^{\text {th }}$ International Conf., $p p$. 5243-5246, Lyon, France, August 2007

[15] M. Strzelecki, J. Certaines and S. Ko. Segmentation of 3D MR Liver Images Using Synchronised Oscillators Network. IEEE, Info. Tech. Conf.,pp.259-263, South Korea, November 2007

[16] C. Platero, M. Gonzalez, M. C. Tobar, J. M. Poncela, J. Sanguino, G. Asensio and E. Santas. Automatic Method To Segment The Liver On MultiPhase MRI. Computer Assisted Radiology and Surgery (CARS), 22nd International Congress and Exhibition, Barcelona, Spain, June 2008

[17] M. Fenchel, S. Thesen and A. Schilling. Reconstructing Liver Shape and Position from MR Image Slices Using an Active Shape Model, Proc. of SPIE,.6914:69144N, 2008

[18] A. Rafiee, H. Masoumi, A. Roosta. Using Neural Network for Liver Detection in Abdominal MRI Images. IEEE Int. Conf. on Signal and Image Proc., pp.21-26, Kuala Lumpur, Malaysia, November 2009

[19] C. Dongxiang, L. Tiankun. Iterative Quadtree Decomposition Segmentation Of Liver MR Images. International Conference on Artificial Intelligence and Computational Intelligence, AICI, 3:527-529, 2009

[20] A. K. Jha, J. J. Rodriguez, R. M. Stephen and A. T. Stopeck. A Clustering Algorithm for Liver Lesion Segmentation of Diffusion Weighted MR Images. IEEE SSIAI, pp.93-96, 2010

[21] O. Gloger, J. Kühn, A. Stanski, H. Völzke and R. Puls. A Fully Automatic Three-Step Liver Segmentation Method on LDA-Based Probability Maps For Multiple Contrast MR Images. International Journal of Magnetic Resonance Imaging, 28(6):882-897, 2010

[22] S. Tang, Y. Wang. MR-Guided Liver Cancer Surgery by Nonrigid Registration. IEEE International Conf. on Medical Image Analysis and Clinical Application (MIACA), pp.113-117, 2010

[23] L. Hermoye, I. Laamari-Azjal, Z. Cao, L. Annet, J. Lerut, B. M. Dawant, B. E. Van Beers. Liver Segmentation In Living Liver Transplant Donors: Comparison of Semiautomatic And Manual Methods. International Journal of Radiology, 234:171-178, 2005

[24] K. Cheng, G. Lixu, W. Jianghua, L. Wei, X. Jianrong. A Novel Level Set Based Shape Prior Method For Liver Segmentation From MRI Images. MIAR2008, LNCS 5128, 150-159, 2008

[25] Y. Shi and W. C. Karl. A Real-Time Algorithm For The Approximation Of Level-Set Based Curve Evolution. IEEE Trans. Image Process., 17:645656, 2008

[26] S. Osher and J. A. Sethian. Fronts Propagating with Curvature-dependent Speed: Algorithms Based on Hamilton-Jacobi Formulation. Journal of Comput. Phys., 79(1):12-49, 1988

[27] C. Li, C. Kao, J. C. Gore and Z. Ding. Minimization Of Region Scalable Fitting Energy For Image Segmentation. IEEE Trans. Imag. Proc, 17(10):1940-1949, 2008

[28] T. Chan and L. Vese. Active Contours Without Edges. IEEE Trans. Image Process., 10(2):266-277, 2001

[29] V. Caselles, F. Catte, T. Coll and F. Dibos. A Geometric Model for Active Contours in Image Processing. Numerische Mathematik, 66(1):1-31, 1993

[30] R. Malladi, J. A. Sethian and B. C. Vemuri. Shape Modeling With Front Propagation: A Level Set Approach. IEEE Transactions on Pattern Analysis and Machine Intelligence, 17(2):158-175, 1995

[31] V. Caselles, R. Kimmel and G. Sapiro. Geodesic Active Contours. Int. Journal of Comput. Vision.,22(1):61-79, 1997

[32] L. Wang, C. Li, Q. Sun, D. Xia and C. Y. Kao. Active Contours Driven by Local and Global Intensity Fitting Energy with Application to Brain MR Image Segmentation. Computerized Medical Imaging and Graphics, 33(7):520-531, 2009

[33] K. Zhang, H. Song and L. Zhang. Active Contours Driven By Local Image Fitting Energy. Pattern Recognition 43:1199-1206, 2010

[34] B. Wang, X. Gao, D. Tao and X. Li. A Unified Tensor Level Set For Image Segmentation. IEEE Trans. Syst.,Man, Cybern. B, Cybern., 40(3):857, 2010

[35] J. An, M. Rousson and Xu C. Г-convergence Approximation to Piecewise Smooth Medical Image Segmentation. MICCAI (2):495-502, 2007

[36] S. Lankton, J. Melonakos, J. Malcolm, S. Dambreville, and A. Tannenbaum. Localized Statistics for DW-MRI Fiber Bundle Segmentation. Computer Vision and Pattern Recognition Workshops, pp. 1-8, Anchorage, AK, June 2008 
[37] C. Li, C. Kao, J. Gore and Z. Ding. Implicit Active Contours Driven By Local Binary Fitting Energy. Proceedings of IEEE Conference on Computer Vision and Pattern Recognition (CVPR2007), pp. 1-7, USA, July 2007

[38] J. Piovano, M. Rousson and T. Papadopoulo. Efficient Segmentation of Piecewise Smooth Images. SSVM2007, LNCS4485, 4485:709-720, 2007

[39] A. Chakraborty and J.S. Duncan. Game-theoretic Integration for Image Segmentation. IEEE Trans. Pattern Anal. Machine Intell. 21(1):12-30, 1999

[40] A.F. Frangi, W.J. Niessen and M. A. Viergever. Three-dimensional Modelling for Functional Analysis of Cardiac Images: A review. IEEE Trans. Med. Imaging 20(1):2-25, 2001

[41] C. Xu, D.L. Pham, and J. L. Prince. Medical Image Segmentation Using Deformable Models. Handbook of Medical Imaging Vol. 3, SPIE Pres, Medical Image Processing and Analysis, pp.129-174, 2000

[42] M. Kass, A. Witkin and D. Terzopoulos. Snakes: Active Contour Models. Int. Journal of Comp. Vis., 1:321-331, 1988

[43] C. Xu and J. L. Prince. Snake, Shapes, And Gradient Vector Flow. IEEE Trans. Image Process., 7(3):359-369, 1998

[44] T. McInerney and D. Terzopoulos. T-snakes: Topologically Adaptive Snakes. Med. Image Anal., 4(2):73-91, 2000

[45] S. C. Zhu and A. Yuille. Region Competition: Unifying Snakes, Region Growing, and Bayes/MDL For Multiband Image Segmentation. IEEE Trans. Pattern Anal. Machine Intell., 18(9):884-900, 1996

[46] G. A. Giraldi, E. Strauss and A. A. Oliveira. A Boundary Extraction Method Based on Dual-T-snakes and Dynamic Programming. Proc. IEEE Comput. Vis. Pattern Recognition, (CVPR), 1:44-49, 2000

[47] C. Xu and J. L. Prince. Generalized Gradient Vector Flow External Forces For Active Contours. Signal Processing, 71(2):131-139, 1998

[48] B. Ostlad and A. Tonp. Encoding of A Priori Information in Active Contour Models. IEEE Trans. Pattern Anal. Machine Intell.,18(9):863-872, 1996

[49] S. D. Fenster and J. R. Kender. Sectored Snakes: Evaluating Learned Energy Segmentations. IEEE Trans. Pattern Anal. Machine Intell., 23(9):1028-1034, 2001

[50] H. Delingette and J. Montagnat. Shape And Topology Constraints On Parametric Active Contours. Comput. Vis. Image Understand., 83:140-171, 2001

[51] B. B. Kimia, A. Tannenbaum and S. W. Zucker. Toward a Computational Theory of Shape: An Overview. Computer Vision-ECCV 90. (Ed. O. Faugeras). Lecture Notes in Computer Science, 427:402-407, 1990

[52] M. Rousson and N. Paragios. Shape Priors For Level Set Representations. Proceedings of the Seventh European Conference on Computer Vision (ECCV'02), 2351:78-92, 2002

[53] N. Paragios and R. Deriche. Geodesic Active Region And Level Set Methods For Supervised Texture Segmentation. Int. Journal of Comput. Vis.,46(3):223-247, 2002

[54] Y. Chen, S. Thiruvenkadam, F. Huang, H. D. Tagare, D. Wilson and E. A. Geiser. On The Incorporation Of Shape Priors Into Geometric Active Contours. Proceedings of the IEEE Workshop on Variational and Level Set Methods, pp. 145-152, Vancouver, Canada, 2001

[55] M. Leventon, E. Grimson and O. Faugeras. Statistical Shape Influence in Geodesic Active Contour. Proc. IEEE Comput. Vis. Pattern Recognit. (CVPR), pp.316-322, Hilton Head Island, South Carolina, USA, 2000

[56] L. A. Vese and T. F. Chan. A Multiphase Level Set Framework For Image Segmentation Using The Mumford And Shah Model. Int. Journal of Comput. Vis., 53(3):271-293, 2002

[57] D. Chopp. Computing Minimal Surfaces Via Level Set Curvature Flow. Journal of Comput. Phys., 106(1):77-91, 1993

[58] D. Peng, B. Merriman, S. Osher, H. Zhao and M. Kang. A PDE-Based Fast Local Level Set Method. Journal of Comput. Phys., 155:410-438, 1999

[59] R. Whitaker. A Level-Set Approach To 3D Reconstruction From Range Data. Int. Journal of Comput. Vis., 29(3):203-231, 1998

[60] R. Goldenberg, R. Kimmel, E. Rivlin and M. Rudzsky. Fast Geodesic Active Contours. IEEE Trans. Image Process., 10(10):1467-1475, 2001

[61]S. K. Weeratunga and C. Kamath. An Investigation of Implicit Active Contours for Scientific Image Segmentation. Video Communications and Image Processing, UCRL-CONF-200711, SPIE Electronic Imaging, San Jose, $C A$, USA, January 2004

[62] K. Boesen, K. Rehm, K. Schaper, S. Stoltzner, R. Woods, E. Luders and D. Rottenberg. Quantitative Comparison Of Four Brain Extraction Algorithms. Neurolmage, 22(3):1255-1261, 2004

[63] D. P. Huttenlocher, G. A. Klanderman and W. J. Rucklidge. Comparing Images Using The Hausdorff Distance. IEEE Transactions on Pattern Analysis and Machine Intelligence, 15(9):850 - 863, 1993

[64] Z. Wang, A. C. Bovik, H. R. Sheikh and E. P. Simoncelli. Image Quality Assessment: From Error Visibility To Structural Similarity., IEEE Transactions on Image Processing, 13(4):600-612, 2004
[65] L. He, Z. Peng, B. Everding, X. Wang, C. Y. Han, K. L. Weiss and W. G. Wee. A Comparative Study of Deformable Contour Methods on Medical Image Segmentation. Image and Vision Computing 26(2):141-163, 2008

[66] E. Goceri. Automatic Kidney Segmentation Using Gaussian Mixture Model on MRI Sequences. Electrical Power Systems and Computers, 99; 2329,2011

[67] Z. Wang, A. C. Bovik, H. R. Sheikh and E. P. Simoncelli. Image Quality Assessment: From Error Visibility To Structural Similarity., IEEE Transactions on Image Processing, 13(4):600-612, 2004 